\title{
Contribution of cancer stem cells to tumor vasculogenic mimicry
}

\author{
Xiao-hong Yao, Yi-fang Ping, Xiu-wu Bian ${ }^{凶}$ \\ Institute of Pathology and Southwest Cancer Center, Southwest Hospital, Third Military Medical University, Chongqing 400038, \\ China \\ Correspondence: bianxiuwu@263.net \\ Received March 13, 2011 Accepted March 26, 2011
}

\begin{abstract}
Vasculogenic mimicry (VM), a newly-defined pattern of tumor blood supply, provides a special passage without endothelial cells and is conspicuously different from angiogenesis and vasculogenesis. The biological features of the tumor cells that form VM remain unknown. Cancer stem cells (CSCs) are believed to be tumorinitiating cells, capable of self-renewal and multipotent differentiation, which resemble normal stem cells in phenotype and function. Recently CSCs have been shown to contribute to VM formation as well as angiogenesis. These findings challenge the previous understanding of the cellular basis of VM formation. In this review, we present evidence for participation of CSCs in VM formation. We also discuss the potential mechanisms and possible interaction of CSCs with various elements in tumor microenvironment niche. Based on the importance of VM in tumor progression, it constitutes a novel therapeutic target for cancer.
\end{abstract}

KEYWORDS cancer stem cell, vasculogenic mimicry, differentiation plasticity, niche, microenvironment

\section{INTRODUCTION}

Development and growth of tumors require sufficient blood supply. It is a widely-accepted paradigm that tumor vasculature is mostly composed of non-malignant endothelial cells originating from pre-existing blood vessels sprouting into tumor mass and recruitment of circulating endothelial progenitor cells (EPCs), which are mediated by angiogenic growth factors produced by host or tumor cells (Dorsam and Gutkind, 2007). However, classical patterns of angiogenesis and vasculogenesis have been challenged by clinical investigation of tumor tissues because tumor vasculature can also be formed by vasculogenic mimicry (VM) (Folberg and Maniotis, 2004).

VM is a vascular-like structure through which tumor tissues nourish themselves, mimicking the pattern of embryonic vascular network. Tumor cells with high degree of differentiation plasticity may contribute to the de novo formation of tumor cell-lined blood channels (Hendrix et al., 2003a). These extracellular matrix-rich vasculogenic tumor cell networks were shown to conduct fluid. An interesting observation was that VM was most frequently observed in the boundary regions between the tumor and surrounding normal tissues (Sun et al., 2004). Thus, VM may also play a role in tumor invasion by supplying immediate nutrition. Furthermore, angiogenesis inhibitors abrogated new vessels formed by human vascular endothelial cells in vitro, while under the same conditions they did not affect tumor cell tube network formation, and even induced the formation of $V M$ as an escape mechanism by tumor tissue for progressive growth (van Der Schaft et al., 2004). Therefore, VM might represent an important survival mechanism contributing to the failure of current antiangiogenic therapy aimed to fully deprive tumors of blood supply (Folkman, 2007). Despite of its clinical importance, the cellular and molecular events underlying the formation of VM are not well understood. Recent discovery of cancer stem cells (CSCs), with the capability of self-renewal and multipotency of differentiation, has stimulated great interest in re-defining tumor initiation and progression (Gao, 2007). However, whether CSC theory can be applied to the formation of tumor cell-associated vasculogenesis, especially in respect to VM, remains unclear. Based on the present findings that most vessels in tumor may be originated from tumor cell themselves through the process of vasculogenesis (Pezzolo et al., 2007), as well as that CSCs were able to serve as precursors of tumor stromal compo- 
nents such as tumor vasculogenic stem/progenitor cells (TVPCs) regulated by signals from microenvironment/niche surrounding these cells (Shen et al., 2008), it is plausible that CSC compartment of a tumor may be involved in VM formation, by differentiating/transdifferentiating into endothelial-like cells. Such a potential function of CSCs might represent one of the mechanisms by which CSCs initiate neoplastic formation and promote tumor progression (Bjerkvig et al., 2005). In this review, we will focus on the possible role of CSCs in VM formation and how the niche surrounding CSCs may affect VM formation.

\section{CURRENT UNDERSTANDING OF TUMOR VM}

In 1999, Maniotis et al. first described VM in aggressive melanoma with tumor cells expressing endothelial phenotype pasted on the surface of the basement membrane in tubular structure (Maniotis et al., 1999). VM in the tumor mass is connected with host vessels for blood supply. Periodic acidSchiff (PAS) stain is commonly utilized to identify VM. PASpositive channels were lined externally by tumor cells, lacking an inner lining of endothelial cells (Hendrix et al., 2001). Although the functionality and contribution of VM channels to circulation was criticized initially, Frenkel et al. (Frenkel et al., 2008) demonstrated blood circulation in VM tube with laser scanning confocal angiography in a patient with a choroidal melanoma. Therefore, VM is a new pattern that provides tumor mass nutrition independent of conventional angiogenesis and vasculogenesis. Zhang et al. proposed three-stage blood supply patterns in tumor, which are VM, mosaic vessels (MV) and endothelium-dependent vessels (Zhang et al., 2006). All the three patterns provide blood supply for tumors. The model proposes that VM is the dominant blood supply pattern in the early stage of tumor growth. Consequently, endothelial cells differentiate and proliferate to maintain expansion of tumor mass, and the mosaic vessels appear as a transitional pattern. In the late stage of tumor growth, endothelium-dependent vessels replace VM and mosaic vessels to become the major pattern of blood supply. Thus, VM may be the main source of blood supply at the early stage of rapid tumor growth. Based on PAS staining, VMs are divided into seven categories: straight channels, parallel straight pattern, parallel straight pattern with cross link, arcs (not closed), arcs with branching, closed loops, and networks (Folberg et al., 2000).

VM has been detected in melanoma, breast carcinoma, prostate carcinoma, ovarian carcinoma, astrocytoma, and Ewing sarcoma, etc (Shirakawa et al., 2002; Sun et al., 2004; Yue and Chen, 2005; Elzarrad et al., 2009). Microarray analysis indicates that VM-positive tumor cells of aggressive melanoma displayed elevated levels of genes associated with undifferentiated embryonic-like phenotype (Hendrix et al., 2003a). Intraperitoneal implantation of human ovarian cancer cell line SKOV3ip showed that the cells, expressing CD31 and factor VIII of vascular endothelial markers, had the plasticity to engage in VM formation in vivo (Su et al., 2008). These findings suggest that the plasticity of cancer cells enables them to mimic the activities of endothelial cells and participate in the process of VM formation. Recent findings of "plastic" endothelial-like phenotype of tumor cells provide additional evidence for the role of tumor cells in VM formation.

There are striking parallels between tumor cells and stem cells: tumors and normal tissue are comprised of phenotypically heterogeneous cell populations, and many characteristics of stem cells, for example stem cell plasticity, are also pertinent to tumor growth (Schatton and Frank, 2008). Cellular plasticity in stem cells may facilitate the formation of primary vascular network during embryonic development. Mesodermal progenitor cells differentiate in situ into endothelial cells that are organized into a primitive network to supply nutrition for the development of early embryo (Carmeliet, 2000). The subsequent remodeling of vascular network into more complex vasculature appears through angiogenesis. These processes are similar to the formation of tumor vasculature and the plasticity of tumor cells may play an important role in the formation of VM.

\section{DIFFERENTIATION PLASTICITY OF CSCS AND VM FORMATION}

CSCs are functionally defined by their capacity to regenerate tumors in xenograft mouse models (Galli et al., 2004; Singh et al., 2004). Similar to normal stem cells, CSCs can reproduce the heterogenous phenotype of the parental cancer from which they were derived in transplantation, reflecting the multipotent differentiation capacity of CSCs. Plasticity defines the capacity of stem cells to either differentiate or transdifferentiate into many cell types (D'Amour and Gage, 2003). During development, multilineage differentiation plasticity is one of the characteristics of embryonic stem cells (Reya et al., 2003). CSCs are characterized by their stem/progenitor properties: selfrenewal and the capability of differentiation into heterogeneous tumor cell population (Clarke et al., 2006). Therefore, the differentiation plasticity of normal stem cells is also a similar property of CSCs. Bian et al. proposed a concept of CSC plasticity (CSCP) in which CSCs possess inducible and reversible properties in self-renewal, multipotent differentiation and invasion. For CSCs, differentiation plasticity refers to the ability of tumor cells to give rise to phenotypically diverse populations including non-tumorigenic cancer cells and stromal cells. In fact, aggressive melanoma cells forming VM appeared to express genes relevant to multiple cellular phenotypes and stem cells including epithelial, endothelial, muscle, neuronal, and other cell types. The multipotent, plastic, and embryonic-like phenotype of these melanoma cells has also been considered as a defined property of putative malignant melanoma stem cells (MMSCs) (Schatton 
A

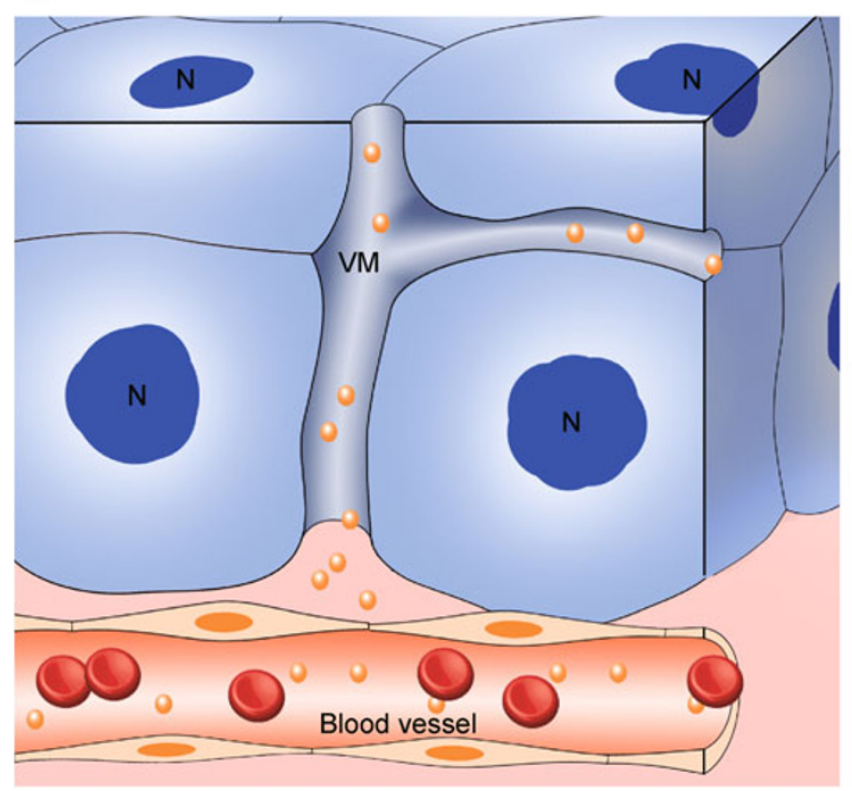

B

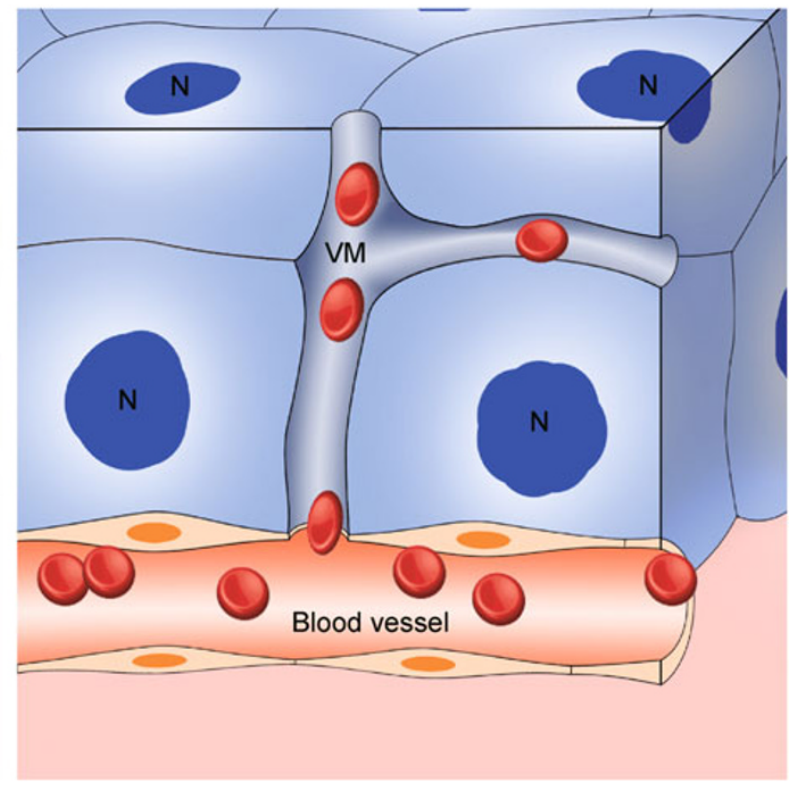

Figure 1. The schematic diagram of CSC participation in VM formation. (A) CSC capacity of VM differentiating/ transdifferentiating and lining up to form branching lumens to provide nutrition for tumor mass. (B) The tubes formed by CSCs progeny extend and merge with vessels from angiogenesis or vascularization, and conduct blood cells. CSC, cancer stem cell; VM, vascular mimicry; $\mathrm{N}$, nucleus.

et al., 2008). Therefore, melanoma stem cells possess the differentiation plasticity (transdifferentiation) and this property may play a critical role in VM. Recently, a transdifferentiative capability has been demonstrated for bone marrow macrophages, which formed VM in multiple myeloma. Thus, at least in melanoma, VM channel was believed to be due to the transdifferentiation of MMSC subset inside the aggressive tumor. In study of breast cancer, CSCs in endothelial differentiating medium were capable of differentiating into endothelial cells, which were able to form both vessels and tumor (Bussolati et al., 2009). It is conceivable that CSCs/ tumor initiating stem cells of solid tumor have the competence of differentiation plasticity, which further supports the hypothesis that CSCs/tumor initiating stem cells possess the properties of normal stem cells important for tumor growth and vascularization.

Evidence for direct involvement of tumor cells in VM formation was also obtained in human neuroblastoma (NB) (Pezzolo et al., 2007). Microvessels formed by MYCNamplified NB tumor cells displayed an open lumen and consistently contained red blood cells, indicating that these vessels were functional. Moreover, these tumor cell-derived vascular endothelium-like cells were different from normal endothelial cells in phenotype and function (Pezzolo et al., 2007). Although the study only tested MYNC-amplified tumor cells, it is possible that formation of tumor-derived endothelial cells is a characteristic feature of a subset of cells in neuroblastoma. In addition to NB, tumor-associated endothelial microvessels are also found in human B-cell lymphomas and multiple myeloma (Rigolin et al., 2006). It is also hypothesized that precancerous stem cells (pCSCs) representing the early stage of developing CSCs may serve as tumor TVPCs capable of differentiating into tumor vasculogenic endothelial cells (Shen et al., 2008). Our recent studies also observed that glioma stem cells (GSCs), isolated from primary glioma sample (Yi et al., 2007) and a human glioblastoma cell line U87 (Yu et al., 2008), are capable of multipotent differentiation. In stem cell medium, such GSCs form spheroids, and in differentiation conditions they form tumor masses that contain fissure and branching lumen as revealed by electron microscopy. Thus, we proposed that CSCs participate in VM formation of tumors. Firstly, CSCs have the capacity of VM aggregation to form a mass in tumorigenic microenvironment. These CSC subsets subsequently differentiate/transdifferentiate and line up to form branching lumens and tubes to provide nutrition for tumor mass, resembling vascular network. Finally, the tubes extend and merge with vessels from angiogenesis or vascularization, and conduct blood cells (Fig. 1).

\section{RELATIONSHIP BETWEEN GSCS, VM AND EPITHELIAL-MESENCHYMAL TRANSITION}

It is believed that epithelial-mesenchymal transition (EMT) is involved in cancer invasion and metastasis. Epithelial cancer cells have the capacity of activating the primal development 
program, thus converting differentiated epithelial tumor cells into de-differentiated cells that possess more mesenchymal feature (Eccles and Welch, 2007). Besides its well-documented contribution to invasion, EMT is also attributed to the formation of secondary location in metastasis sites by conferring self-renewal capability to disseminated cancer cells (Mani et al., 2008; Morel et al., 2008). Recent studies report that EMT is associated with the acquisition of stem-celllike characteristics. Induction of EMT in immortalized human mammary epithelial cells resulted in the expression of stem cell markers, the gain of mesenchymal behavior, and phenotypes associated with CSCs (Mani et al., 2008). The connection between EMT and cellular stemness is further supported by the finding that Twist1, an important regulator of EMT, directly regulated the stemness factor Bmil which is necessary for self-renewal of stem cells (Yang et al., 2010). Constitutive overexpression of Twist1 and Bmil in tumor cell lines caused acquisition of EMT characteristics as well as the induction of stem cell markers and enhancement of tumor initiating capability. In agreement with this finding, the induction of an EMT by ectopic expression of Twist transcription factor has been reported to have generated cancer stem cell properties in human breast cancer cells (Mani et al., 2008).

Furthermore, there is evidence showing that EMT contributes to the formation of VM in hepatocellular carcinoma (HCC) (Sun et al., 2010). CSCs in HCC expressed higher level of EMT regulators, such as Twist and Snail ( $\mathrm{Na}$ et al., 2011). Upregulation of Twist1 in HCC significantly enhanced cell motility, invasiveness and VM formation in 3D culture system as well as expression of VM-associated molecules such as VE-cadherin (Sun et al., 2010). These findings indicate a logical relationship between CSCs, EMT and gain of properties characteristic of VM forming cells. The VM formation involving tumor cells mimics endothelial cells consisting of a type of mesenchymal cell, similar to the EMT process. VM forming cells in tumors are characterized by the expression of various factors responsible for mediating the process of EMT at the molecular level. Thus, the EMT mechanism may be involved in the VM formation by CSCs.

\section{MICROENVIRONMENTAL NICHE AS A REGULATOR OF VM FORMATION}

The vasculogenesis and/or angiogenesis, which are necessary for tumor development and progression, involve the interaction of tumor and other cell types in the microenvironment or niche (Elias and Dias, 2008). A pertinent role of the microenvironment in VM formation has been demonstrated in melanoma (Hendrix et al., 2003b). Collagen matrices preconditioned by aggressive melanoma cells capable of forming VM induced lesser aggressive melanoma cells, which are initially unable to form VM to express vasculogenic genes and to form VM in vitro. These observations illustrate the remarkable influence of microenvironment on the phenotype of tumor cells and provide a new perspective for the formation of $\mathrm{VM}$, in which factors secreted by tumor cells or other niche components in the microenvironment play a critical role in cancer cell plasticity, including dedifferentiation and transdifferentiation. In addition, the microenvironmental niche has been demonstrated to support normal stem cells in early coculture and transplantation studies (Schofield, 1978). One of the mechanisms through which microenvironmental niche determines normal stem cell fate is the control of symmetric (producing two identical daughter cells) versus asymmetric (producing one identical and one differentiated cell) division (Morrison and Kimble, 2006). CSCs, like normal stem cells, also depend on interaction with physiologically differentiated cell types or on non-tumorigenic cancer cell populations in the same tumor microenvironment to sustain their features and destiny (Scadden, 2006). Tumor environment creates a niche favoring the survival, proliferation, and differentiation of CSCs. CSCs utilize a specialized microenvironment/niche termed tumor stroma, consisting of a combination of different cell lineages, i.e. epithelial, vascular, fat, glial, fibroblast, immune cells along with extracellular matrix, enzymes, and other secreted molecules produced by these cells (Vermeulen et al., 2008). It has been demonstrated that endothelial cells surrounding CSCs appear to directly generate specific microvasculature niche and/or secrete factors that promote the formation and/or maintenance of brain CSCs (Calabrese et al., 2007). Critical signaling molecules, such as bone morphogenetic proteins (BMPs) derived from the niche that govern embryonic vascular development, have been linked to melanoma cell-driven vasculogenesis, i.e. VM (Rothhammer et al., 2007). In human glioblastomas, BMP4-BMPR1a signaling pathway regulates the differentiation and proliferation of CSC population (Piccirillo et al., 2006; Nakano et al., 2008). Based on these findings, it is plausible that the niche of CSC compartment controls the differentiation plasticity of CSCs, which is responsible for tumor vasculogenesis including VM formation.

In addition to being conditioned by niche compents, CSCs may also reciprocally influence the niche through secretion of autocrine and/or paracrine factors or through direct cell-cell contact to benefit the maintenance of their stemness including self-renewal, multipotent differentiation, and tumor-initiation. We and others have suggested that CSCs from U87 glioblastoma cell line and primary brain tumors secrete higher levels of vascular endothelial growth factor (VEGF) than their non-tumorigenic counterpart cells that promoted the formation of tumor blood vessels (Bao et al., 2006; Yao et al., 2008). In breast cancer model, VEGF induces CSCs to express endothelial markers in vitro and incorporate in tumor vasculature in vivo (Bussolati et al., 2009). Accumulating evidence shows in addition to the molecules of vascular endothelial (VE)-cadherin, laminin 5 Y2 chain, and vascular endothelial growth factor receptor-2 (VEGFR-2), the angiogenic factors, including VEGF, angiogenin-1, and ephrinA1, 
also played a critical role in the formation of $\mathrm{VM}$ by tumor cells (Basu et al., 2006). In healthy subjects, stimulated by VEGF, cells of monocyte lineage (other mesodermal-derived cells) present an endothelial phenotype, and form a functional capillary-like mesh permeable by blood cells, recapitulating embryonic vasculogenesis. VEGF also stimulated macrophages of patients with active multiple myeloma to undergo phenotypic and functional adaptation, expressed by the markers of endothelial cells, i.e. VE-cadherin, VEGFR-2, and FVIII-RA, and retain their own CD14 and CD68 markers, and these cells can form vessel-like structures on the Matrigel surface. Therefore, VEGF can induce these macrophages to transdifferentiate into endothelial-like cells to form VM, which functionally, phenotypically and morphologically were similar to endothelial cells, yet maintained the expression of macrophage markers. Thus, VEGF in the niche, which may be derived mainly from CSCs, directly influences the phenotype of CSCs and promotes CSC-associated VM formation.

There are three factors manipulating the formation of VM channel: the plasticity of VM-associated tumor cells, remodeling of extracellular matrix, and the connection of VM with host microcirculation (Fujimoto et al., 2006). The remodeling of extracellular matrix provided the space needed for VM and is regulated by matrix metalloproteinase (MMP) (Stevens et al., 2009). Matrix MMP-9 and MMP-2 play a critical role during the formation of VM in aggressive melanoma. Our recent results showed that the expression of MMP-9 and MMP-2 is upregulated in GSCs derived from U87 cell line (Yu and Bian, 2009). The formation of VM also involves migration of VM-derived tumor cells. We observed that migration associated molecules, including two G-protein coupled chemoattractant receptors formylpeptide receptor (FPR) and CXC chemokine receptor-4 (CXCR4) were overexpressed in GSCs isolated from human glioblastoma and U87 cell line (Ping et al., 2007; Yao et al., 2008). FPR and CXCR4 expressed on GSCs, when activated by corresponding agonists, mediate directional migration, calcium mobilization, and production of VEGF by GSCs. Our recent observations further suggest that activation of CXCR4 on GSCs elicits phosphoinositide 3-kinase (PI3K) pathway (Ping et al., 2011) which is an important regulator of VM through MMP-2 (Hess et al., 2003). The relationship between CSCs and VM formation through stimulatory signals in the niche is important for differentiation plasticity of CSCs (Ping and Bian, 2011). Based on the existing observations, it is conceivable that CSCs take part in the VM formation through autocrine and/or paracrine manner thereby establishing a vessel niche suitable to protect and nourish CSCs. Therefore, VM-targeted therapies should be a new strategy aimed at eliminating CSCs.

\section{VM-TARGETED THERAPEUTIC STRATEGY: NEW PERSPECTIVES}

CSCs are considered as the root of tumor initiation, metastasis, and reoccurrence. If CSCs are proven to be critical for VM formation, there will be significant implications in the design of novel anti-tumor therapies. As discussed earlier, VM is the dominant blood supply pattern in the early stage of tumor formation and CSCs are capable of differentiating/transdifferentiating and lining up to form branching lumens and tubes, a process resembling the formation of VM. Traditional anti-angiogenesis drugs, such as angiostatin and endostatin, which target normal endothelial cells, have little effect on VM due to the absence of normal endothelial cells (Hillen and Griffioen, 2007). In contrast, VEGF-specific inhibitor Bevacizumab can conspicuously decrease the number of self-renewing cancer cells from orthotopic models of medulloblastoma and glioma, resulting in tumor growth arrest. Direct evidence was obtained from aggressive melanoma that LY294002, a specific inhibitor of $\mathrm{PI} 3 \mathrm{~K}$, inhibited the ability of undifferentiated embryonic melanoma cells to engage in VM on three-dimensional type I collagen matrices (Hess et al., 2003). Furthermore, the unique structure of VM channels, in which tumor cells line up the inner surface, directly exposes tumor cells to blood vessel and facilitates the metastasis of tumor cells. VM frequently is seen in the regions between the tumor and surrounding normal tissues, and associated with poor prognosis in clinical patients. Therefore, VM-targeted therapies may destroy the niche that maintains CSCs, block the metastasis passage of tumor cells, and reduce the recurrence of cancer.

\section{ACKNOWLEDGEMENTS}

This study was supported by grants from the National Basic Research Program of China (973 Program) (Grant No. 2010CB529403) and National Natural Science Foundation of China (Grant Nos. 30800421 , 30725035 and 30930103).

\section{ABBREVIATIONS}

BMPs, bone morphogenetic proteins; CSCs, cancer stem cells; CSCP, CSC plasticity; EMT, epithelial-mesenchymal transition; EPCs, endothelial progenitor cells; MMP, matrix metalloproteinase; MMSCs, malignant melanoma stem cells; MV, mosaic vessels; pCSCs, precancerous stem cells; TVPCs, tumor vasculogenic stem/ progenitor cells; VEGF, vascular endothelial growth factor; VM, vasculogenic mimicry

\section{REFERENCES}

Bao, S., Wu, Q., Sathornsumetee, S., Hao, Y., Li, Z., Hjelmeland, A. B., Shi, Q., McLendon, R.E., Bigner, D.D., and Rich, J.N. (2006). Stem cell-like glioma cells promote tumor angiogenesis through vascular endothelial growth factor. Cancer Res 66, 7843-7848.

Basu, G.D., Liang, W.S., Stephan, D.A., Wegener, L.T., Conley, C.R., Pockaj, B.A., and Mukherjee, P. (2006). A novel role for cyclooxygenase-2 in regulating vascular channel formation by human breast cancer cells. Breast Cancer Res 8, R69.

Bjerkvig, R., Tysnes, B.B., Aboody, K.S., Najbauer, J., and Terzis, A. J.A. (2005). Opinion: the origin of the cancer stem cell: current controversies and new insights. Nat Rev Cancer 5, 899-904. 
Bussolati, B., Grange, C., Sapino, A., and Camussi, G. (2009). Endothelial cell differentiation of human breast tumour stem/ progenitor cells. J Cell Mol Med 13, 309-319.

Calabrese, C., Poppleton, H., Kocak, M., Hogg, T.L., Fuller, C., Hamner, B., Oh, E.Y., Gaber, M.W., Finklestein, D., Allen, M., et al. (2007). A perivascular niche for brain tumor stem cells. Cancer Cell 11, 69-82.

Carmeliet, P. (2000). Mechanisms of angiogenesis and arteriogenesis. Nat Med 6, 389-395.

Clarke, M.F., Dick, J.E., Dirks, P.B., Eaves, C.J., Jamieson, C.H., Jones, D.L., Visvader, J., Weissman, I.L., and Wahl, G.M. (2006) Cancer stem cells-perspectives on current status and future direction: AACR Workshop on cancer stem cells. Cancer Res 66, 9339-9344.

D'Amour, K.A., and Gage, F.H. (2003). Genetic and functional differences between multipotent neural and pluripotent embryonic stem cells. Proc Natl Acad Sci U S A 100, 11866-11872.

Dorsam, R.T., and Gutkind, J.S. (2007). G-protein-coupled receptors and cancer. Nat Rev Cancer 7, 79-94.

Eccles, S.A., and Welch, D.R. (2007). Metastasis: recent discoveries and novel treatment strategies. Lancet 369, 1742-1757.

Elias, A.P., and Dias, S. (2008). Microenvironment changes (in pH) affect VEGF alternative splicing. Cancer Microenviron 1, 131-139.

Elzarrad, K., Haroon, A., Reed, D., and Al-Mehdi, A.B. (2009). Early incorporated endothelial cells as origin of metastatic tumor vasculogenesis. Clin Exp Metastasis 26, 589-598.

Folberg, R., Hendrix, M.J., and Maniotis, A.J. (2000). Vasculogenic mimicry and tumor angiogenesis. Am J Pathol 156, 361-381.

Folberg, R., and Maniotis, A.J. (2004). Vasculogenic mimicry. APMIS 112, 508-525.

Folkman, J. (2007). Angiogenesis: an organizing principle for drug discovery? Nat Rev Drug Discov 6, 273-286.

Frenkel, S., Barzel, I., Levy, J., Lin, A.Y., Bartsch, D.U., Majumdar, D., Folberg, R., and Pe'er, J. (2008). Demonstrating circulation in vasculogenic mimicry patterns of uveal melanoma by confocal indocyanine green angiography. Eye (Lond) 22, 948-952.

Fujimoto, A.O.H., Onodera, H., Mori, A., Nagayama, S., Yonenaga, Y., and Tachibana, T. (2006). Tumour plasticity and extravascular circulation in ECV304 human bladder carcinoma cells. Anticancer Res 26, 59-69.

Galli, R., Binda, E., Orfanelli, U., Cipelletti, B., Gritti, A., De Vitis, S., Fiocco, R., Foroni, C., Dimeco, F., and Vescovi, A. (2004). Isolation and characterization of tumorigenic, stem-like neural precursors from human glioblastoma. Cancer Res 64, 7011-7021.

Gao, J.X. (2007). Cancer stem cells: the lessons from precancerous stem cells. J Cell Mol Med 58, 267-284.

Hendrix, M.J., Seftor, E.A., Hess, A.R., and Seftor, R.E. (2003a). Vasculogenic mimicry and tumour-cell plasticity: lessons from melanoma. Nat Rev Cancer 3, 411-421.

Hendrix, M.J., Seftor, E.A., Kirschmann, D.A., Quaranta, V., and Seftor, R.E. (2003b). Remodeling of the microenvironment by aggressive melanoma tumor cells. Ann N Y Acad Sci 995, 151-161.

Hendrix, M.J., Seftor, E.A., Meltzer, P.S., Gardner, L.M., Hess, A.R., Kirschmann, D.A., Schatteman, G.C., and Seftor, R.E. (2001). Expression and functional significance of VE-cadherin in aggressive human melanoma cells: role in vasculogenic mimicry. Proc Natl Acad Sci U S A 98, 8018-8023.
Hess, A.R., Seftor, E.A., Seftor, R.E., and Hendrix, M.J. (2003). Phosphoinositide 3-kinase regulates membrane Type 1-matrix metalloproteinase (MMP) and MMP-2 activity during melanoma cell vasculogenic mimicry. Cancer Res 63, 4757-4762.

Hillen, F., and Griffioen, A.W. (2007). Tumour vascularization: sprouting angiogenesis and beyond. Cancer Metastasis Rev 26, 489-502.

Mani, S.A., Guo, W., Liao, M.J., Eaton, E.N., Ayyanan, A., Zhou, A.Y., Brooks, M., Reinhard, F., Zhang, C.C., Shipitsin, M., et al. (2008). The epithelial-mesenchymal transition generates cells with properties of stem cells. Cell 133, 704-715.

Maniotis, A.J., Folberg, R., Hess, A., Seftor, E.A., Gardner, L.M.G., Pe'er, J., Trent, J.M., Meltzer, P.S., and Hendrix, M.J.C. (1999). Vascular channel formation by human melanoma cells in vivo and in vitro: vasculogenic mimicry. Am J Pathol 155, 739-752.

Morel, A.P., Lièvre, M., Thomas, C., Hinkal, G., Ansieau, S., and Puisieux, A. (2008). Generation of breast cancer stem cells through epithelial-mesenchymal transition. PLoS One 3, e2888.

Morrison, S.J., and Kimble, J. (2006). Asymmetric and symmetric stem-cell divisions in development and cancer. Nature 441, 1068-1074.

Na, D.C., Lee, J.E., Yoo, J.E., Oh, B.K., Choi, G.H., and Park, Y.N. (2011). Invasion and EMT-associated genes are up-regulated in $B$ viral hepatocellular carcinoma with high expression of CD133human and cell culture study. Exp Mol Pathol 90, 66-73.

Nakano, I., Saigusa, K., and Kornblum, H.I. (2008). BMPing off glioma stem cells. Cancer Cell 13, 3-4.

Pezzolo, A., Parodi, F., Corrias, M.V., Cinti, R., Gambini, C., and Pistoia, V. (2007). Tumor origin of endothelial cells in human neuroblastoma. J Clin Oncol 25, 376-383.

Piccirillo, S.G., Reynolds, B.A., Zanetti, N., Lamorte, G., Binda, E., Broggi, G., Brem, H., Olivi, A., Dimeco, F., and Vescovi, A.L. (2006). Bone morphogenetic proteins inhibit the tumorigenic potential of human brain tumour-initiating cells. Nature 444 , 761-765.

Ping, Y.F., and Bian, X.W. (2011). Cancer stem cells switch on tumor neovascularization. Curr Mol Med 11, 69-75.

Ping, Y.F., Yao, X.H., Bian, X.W., Chen, J.H., Zhang, R., Yi, L., and Zhou, Z.H. (2007). Activation of CXCR4 in human glioma stem cells promotes tumor angiogenesis. Zhonghua Bing Li Xue Za Zhi 36, 179-183.

Ping, Y.F., Yao, X.H., Jiang, J.Y., Zhao, L.T., Yu, S.C., Jiang, T., Lin, M. C., Chen, J.H., Wang, B., Zhang, R., Cui, Y.H., Qian, C., Wang, J. M., and Bian, X.W. (2011). The chemokine CXCL12 and its receptor CXCR4 promote glioma stem cell-mediated VEGF production and tumor angiogenesis via PI3K/AKT signaling. J Pathol doi: 10.1002/path.2908.

Reya, T., Duncan, A.W., Ailles, L., Domen, J., Scherer, D.C., Willert, K., Hintz, L., Nusse, R., and Weissman, I.L. (2003). A role for Wnt signalling in self-renewal of haematopoietic stem cells. Nature 423 , 409-414.

Rigolin, G.M., Fraulini, C., Ciccone, M., Mauro, E., Bugli, A.M., De Angeli, C., Negrini, M., Cuneo, A., and Castoldi, G. (2006). Neoplastic circulating endothelial cells in multiple myeloma with $13 q 14$ deletion. Blood 107, 2531-2535.

Rothhammer, T., Bataille, F., Spruss, T., Eissner, G., and Bosserhoff, A.K. (2007). Functional implication of BMP4 expression on angiogenesis in malignant melanoma. Oncogene 26, 4158-4170. 
Scadden, D.T. (2006). The stem-cell niche as an entity of action. Nature 441, 1075-1079.

Schatton, T., and Frank, M.H. (2008). Cancer stem cells and human malignant melanoma. Pigment Cell Melanoma Res 21, 39-55.

Schatton, T., Murphy, G.F., Frank, N.Y., Yamaura, K., Waaga-Gasser, A.M., Gasser, M., Zhan, Q., Jordan, S., Duncan, L.M., Weishaupt, C., et al. (2008). Identification of cells initiating human melanomas. Nature 451, 345-349.

Schofield, R. (1978). The relationship between the spleen colonyforming cell and the haemopoietic stem cell. Blood Cells 4, 7-25.

Shen, R., Ye, Y., Chen, L., Yan, QT., Barsky, SH., Gao, JX. (2008) Precancerous stem cells can serve as tumor vasculogenic progenitors. PLoS ONE 3, e1652.

Shirakawa, K., Wakasugi, H., Heike, Y., Watanabe, I., Yamada, S., Saito, K., and Konishi, F. (2002). Vasculogenic mimicry and pseudo-comedo formation in breast cancer. Int J Cancer 99, 821-828.

Singh, S.K., Hawkins, C., Clarke, I.D., Squire, J.A., Bayani, J., Hide, T., Henkelman, R.M., Cusimano, M.D., and Dirks, P.B. (2004). Identification of human brain tumour initiating cells. Nature 432 , 396- 401.

Stevens, A.P., Spangler, B., Wallner, S., Kreutz, M., Dettmer, K., Oefner, P.J., and Bosserhoff, A.K. (2009). Direct and tumor microenvironment mediated influences of $5^{\prime}$-deoxy-5'-(methylthio) adenosine on tumor progression of malignant melanoma. $\mathrm{J}$ Cell Biochem 106, 210-219.

Su, M., Feng, Y.J., Yao, L.Q., Cheng, M.J., Xu, C.J., Huang, Y., Zhao, Y.Q., Jiang, H., Su, M., Feng, Y.J., et al. (2008). Plasticity of ovarian cancer cell SKOV3ip and vasculogenic mimicry in vivo. Int $\mathrm{J}$ Gynecol Cancer 18, 476-486.

Sun, B., Zhang, S., Zhao, X., Zhang, W., and Hao, X. (2004). Vasculogenic mimicry is associated with poor survival in patients with mesothelial sarcomas and alveolar rhabdomyosarcomas. Int J Oncol 25, 1609-1614.

Sun, T., Zhao, N., Zhao, X.L., Gu, Q., Zhang, S.W., Che, N., Wang, X. H., Du, J., Liu, Y.X., and Sun, B.C. (2010). Expression and functional significance of Twist1 in hepatocellular carcinoma: its role in vasculogenic mimicry. Hepatology 51, 545-556.

van der Schaft, D.W., Seftor, R.E., Seftor, E.A., Hess, A.R., Gruman, L.M., Kirschmann, D.A., Yokoyama, Y., Griffioen, A.W., and Hendrix, M.J. (2004). Effects of angiogenesis inhibitors on vascular network formation by human endothelial and melanoma cells. J Natl Cancer Inst 96, 1473-1477.

Vermeulen, L., Todaro, M., de Sousa Mello, F., Sprick, M.R., Kemper, K., Perez Alea, M., Richel, D.J., Stassi, G., and Medema, J.P. (2008). Single-cell cloning of colon cancer stem cells reveals a multi-lineage differentiation capacity. Proc Natl Acad Sci U S A 105, 13427-13432.

Yang, M.H., Hsu, D.S., Wang, H.W., Wang, H.J., Lan, H.Y., Yang, W. H., Huang, C.H., Kao, S.Y., Tzeng, C.H., Tai, S.K., et al. (2010). Bmi1 is essential in Twist1-induced epithelial-mesenchymal transition. Nat Cell Biol 12, 982-992.

Yao, X.H., Ping, Y.F., Chen, J.H., Xu, C.P., Chen, D.L., Zhang, R., Wang, J.M., and Bian, X.W. (2008). Glioblastoma stem cells produce vascular endothelial growth factor by activation of a Gprotein coupled formylpeptide receptor FPR. J Pathol 215, 369-376.

Yi, L., Zhou, Z.H., Ping, Y.F., Chen, J.H., Yao, X.H., Feng, H., Lu, J.Y., Wang, J.M., and Bian, X.W. (2007). Isolation and characterization of stem cell-like precursor cells from primary human anaplastic oligoastrocytoma. Mod Pathol 20, 1061-1068.

Yu, S.C., and Bian, X.W. (2009). Enrichment of cancer stem cells based on heterogeneity of invasiveness. Stem Cell Rev 5, 66-71.

Yu, S.C., Ping, Y.F., Yi, L., Zhou, Z.H., Chen, J.H., Yao, X.H., Gao, L., Wang, J.M., and Bian, X.W. (2008). Isolation and characterization of cancer stem cells from a human glioblastoma cell line U87. Cancer Lett 265, 124-134.

Yue, W.Y., and Chen, Z.P. (2005). Does vasculogenic mimicry exist in astrocytoma? J Histochem Cytochem 53, 997-1002.

Zhang, S., Guo, H., Zhang, D., Zhang, W., Zhao, X., Ren, Z., and Sun, B. (2006). Microcirculation patterns in different stages of melanoma growth. Oncol Rep 15, 15-20. 